\title{
Real-Time Stereo Matching Using Memory-Efficient Belief Propagation for High-Definition 3D Tele-Presence Systems
}

\author{
Jesús M. Pérez ${ }^{1}$, Pablo Sánchez ${ }^{1}$, and Marcos Martinez² \\ ${ }^{1}$ University of Cantabria, Av. Los Castros S/N, 39005 Santander, Spain \\ \{chuchi, sanchez\}@teisa.unican.es \\ ${ }^{2}$ DS2, Charles Robert Darwin 2, Parc Tecnológic, 46980, Paterna, Valencia, Spain \\ marcos.martinez@ds2.es
}

\begin{abstract}
High-definition 3D video is one of the features that the next generation of telecommunication systems is exploring. Real-time requirements limit the execution time of stereo-vision techniques to 40-60 milliseconds. Classical belief propagation algorithms (BP) generate high quality depth maps. However, the huge number of required memory accesses limits their applicability in real systems.

This paper proposes a real-time (latency inferior to 40 millisenconds) highdefinition (1280x720) stereo matching algorithm using belief propagation. There are two main contributions. The first one is an improved BP algorithm with occlusion, potential errors and texture-less handling that outperforms classical multi-grid bipartite-graph BP while reducing the number of memory accesses. The second one is an adaptive message compression technique with low performance penalty that greatly reduces the memory traffic. The combination of these techniques outperforms classical BP by about $6.0 \%$ while reducing the memory traffic by more than $90 \%$.
\end{abstract}

Keywords: Stereo-vision, Belief propagation, High-Definition, Real-Time, FPGA.

\section{Introduction}

In current telecommunication systems, the participants normally do not have the feeling of being physically together in one place. In order to improve the immersive faceto-face experience, tele-presence systems are starting to include 3D video and depth estimation capabilities. A typical requirement for these systems [1] includes high definition (at least 1280x720 pixels), good immersive feeling (more than 80 disparity levels) and low latency (depth estimation in less than 40 milliseconds).

Stereo matching using Belief Propagation (BP) is one of the most effective depth estimation techniques, covering the first positions in the Middlebury rankings. Most

\footnotetext{
* This work has been partially supported by the Spanish CDTI under project CENIT-VISION 2007-1007 and the CICYT under TEC2008-04107.
} 
of the work using BP is based on the global approach presented in [3], because it converges faster and reduces the memory requirements. However, the execution time of this algorithm in a CPU cannot satisfy real-time (RT) requirements with highdefinition (HD) images. Other works [4] are focused on local or semi-global methods. They reduce the execution time, but they normally lose performance. There are some $\mathrm{BP}$ algorithms that have been implemented in GPUs although they have limited performance, working with low-resolution images and a small number of disparity levels [5][6]. Finally, several FPGA-based implementations of BP algorithms have been proposed. In [7], an approach that works with low-resolution images and 16 depth levels is proposed. In [2], a RT architecture is presented. However, they work with only 16 disparity levels and a phase-based depth estimation algorithm, which performs worse than BP-based algorithms. A recent publication on FPGA [20] is also focused on implementing BP-based stereo matching on RT. However, our proposal outperforms [20] in three key aspects: first, it perform 1843.2 million disparity estimations per second (obtained as "width*height*fps*Disparity_labels"), being three time faster than [20]. Secondly, their results are close to the BP-M algorithm, which shows poorer results than our proposal. Finally, our proposal can be implemented in a FPGA, while the one in [20] is an ASIC (very expensive and ad-hoc solution).

With recent hardware advances, memory bandwidth has become a more performance-limiting factor than the total number of algorithm operations. To confront this problem, the image is split into several unconnected regions in [8]. The main drawback for RT applications is that the size of the regions is normally very small and this greatly reduces performance.

Here, we present a stereo matching algorithm based on BP. It includes occlusion, potential error and texture-less region handling. Several techniques have been used in stereo matching for occlusion handling [9]. A simple method of detecting occlusion is the cross-checking technique [10]. Other occlusion-handling approaches generate better results [11] but they double the computational complexity. Some other techniques have improved depth estimation in texture-less areas [12]. However, they work with low-resolution images, 48 disparity levels and they do not satisfy RT requirements. Other approaches try to reduce potential error [13], but they work with medium-resolution images, $14.7 \mathrm{fps}$ and 40 disparity levels.

In this paper, we propose a global approach based on a double serial BP. A recently presented work [14] also uses a two-step depth estimation algorithm, although with a local approach. Moreover, it does not comply with RT and HD requirements. Some proposals [15] use several BP modules and show better performance than ours. However, the time they spend to obtain a small image disparity map is 250 times the time we use to obtain a HD disparity map. On the other hand, some proposals have concentrated on reducing the number of messages in the BP [16][17] or on compressing the messages to reduce memory [18]. However, they are not able to meet HD, RT constraints and good results.

The system described here presents a BP architecture that complies with actual tele-presence system requirements [1]. The proposal includes two main contributions:

1. It splits the algorithm into two BPs that work serially. Between the two blocks, a new data-cost is calculated based on a pixel classification. This classification identifies occlusions, potential-error, texture-less and reliable pixels. This contribution 
improves the single BP results while reducing the number of memory accesses for HD and RT systems (250 times faster than [15]).

2. It defines an adaptive message compression technique to reduce memory traffic with little performance penalty. It provides better balance between performance, simplicity and implementation than [16][17][18] . Moreover, [18] shows some limitations: the message compression used in [18] is not lineal, which means it has to uncompress, operate and compress again. In contrast, our proposal operates with compressed messages. Moreover, as was pointed out in [20], in [18] they assume data to be stored with floating point precision, but if the data precision is 8bit, only 30-50\% compression rate can be achieved. Our proposal achieves more than $70 \%$.

The remainder of this paper is organized as follows. In Section 2, we comment the requirements of the tele-presence system. In Section 3, we discuss the double BP with occlusion, error and texture-less handling methods, as well as the compression technique used to meet the memory access requirements. Finally, we present the experimental results and conclusions in Section 4 and 5.

\section{System Requirements}

The tele-presence system, which is developed in [1] must satisfy the following constrains:

1. Real-time system with low latency: the depth-estimation processing time is limited to about 40 milliseconds. This requirement is essential to provide presence feeling.

2. High resolution: the image size is $1280 \times 720$ pixels. At this resolution, the cameras have a maximum frame rate of $30 \mathrm{fps}$.

3. Immersion feeling: in order to obtain a life-like 3D model, at least 80 disparity levels seems to be needed. Additionally, a high-quality depth-estimation algorithm (i.e. Belief Propagation) is necessary.

4. Memory bandwidth of the hardware platform: an actual high-performance platform (for example, a commercial FPGA-based ASIC-prototyping board) has a limited maximum external-memory bandwidth (about $153 \mathrm{~Gb} / \mathrm{seg}$ in the case of the paper reference platform [19]).

As far as the authors know, there are no previous works that can satisfy all these requirements.

\section{Proposed System Architecture}

In order to use reference [3]'s algorithm in a real system, several parameters have to be defined. In this work we have assumed that the minimum number of iterations and levels needed to cover section 2 requirements is 7 . The algorithm variables are quantified using 16 bits and the number of disparity levels is set to 80 . The linear truncated model [3] was chosen for the messages as it presents a good balance between edge information and noise information. With these parameters the BP-based technique presented in [3] satisfies the quality constraint (point 3) in the previous section, despite 
edge error, occlusions and texture-less region flaws. However, it cannot satisfy the RT and memory bandwidth restrictions (points 1 and 4). This algorithm will be referred to as classical BP.

One the most restrictive parameters is the number of external memory accesses. The actual high-performance platform, which is used as the hardware reference model in this work [19], could support up to 6 DDR2-400 memories with 64 bits per memory data bus. The maximum number of memory accesses that a depth estimation algorithm can perform in this platform is about 384 million. Two parameters have been taken into account to obtain this limit: the algorithm variables are quantified with 16 bits and the estimation time is less than 40 milliseconds. However, if the classical BP algorithm is analyzed with the section 3 parameters, the total number of required memory accesses will be 2881 million. Thus, the system is far from being implementable in an actual high-performance platform and it would require a reduction in the number of accesses by almost $90 \%$.

In order to handle occlusions, potential-errors and texture-less regions that degrade the performance of the classical approach, the proposal is to split the BP algorithm into two separate BP blocks. Between them, a new module (Occlusion, Error and texture-less handling module, $\mathrm{OE}$ ) classifies the pixels into four categories. Additionally, this module will recalculate the values of the cost function taking into account the pixel category. Hereinafter, this algorithm will be denoted as Real-time HighDefinition Belief Propagation (RT-HD BP). It performs the following steps:

1. Read left and right images and compute data-cost

2. Iterative BP (BP1) over all the pixels

3. Output: for each pixel, send to the output:

a) Minimum disparity label of the left-image depth map.

b) Third minimum disparity label of the left-image depth map.

c) Minimum disparity label of the right-image depth map.

4. Classify pixels into reliable, occlusion, error and texture-less (OE Module)

5. Calculate new data-cost based on previous classification (OE Module)

6. Iterative BP (BP2) only over non-reliable pixels

7. Output: for each pixel, send to the output:

- Minimum disparity label of the left depth map (final result).

The aim of BP1 is to provide the OE module with enough information to classify the image pixels. A very important advantage of the proposed technique is that this classification can be obtained with a relatively low number of iterations. After the pixel classification has been obtained, the second BP (BP2) generates the final depth map with a reduced number of iterations. Moreover, it also saves memory traffic, performing message passing only on non-reliable pixels (about $20 \%$ of the pixels).

It might seem that the complexity and memory bandwidth requirements of the proposed technique could double the classical BP (there are 2 BP blocks, steps 2 and 6). However, the BP1 and BP2 blocks can be implemented in the same hardware module, as they have exactly the same architecture. Moreover, the total number of memory accesses is reduced with respect to classical BP. In table 1, the number of iterations for each level in RT-HD and classical BP are presented. In classical BP, the number 
of iterations is constant, but in RT-HD BP it changes with the level. Table 1 presents the total number of BP1 and BP2 iterations per level.

Even though the number of iterations is higher in the first levels (6 to 3), the algorithm reduces the iterations in the last level and this minimizes the total number of memory accesses: the classical BP algorithm needs 9.33x accesses while the RT-HD BD needs only $7.47 x$ accesses $(19.89 \%$ less memory traffic). The parameter ' $x$ ' is a function of the image size and disparity levels.

Table 1. Relation between memory accesses and levels

\begin{tabular}{|c|c|c|c|c|c|c|c|}
\hline Level & 0 & 1 & 2 & 3 & 4 & 5 & 6 \\
\hline Classical BP iterations & 7 & 7 & 7 & 7 & 7 & 7 & 7 \\
\hline RT-HD BP iterations & 5 & 7 & 7 & 14 & 14 & 14 & 14 \\
\hline Memory accesses per iteration & $\mathrm{x}$ & $\mathrm{x} / 4$ & $\mathrm{x} / 16$ & $\mathrm{x} / 64$ & $\mathrm{x} / 264$ & $\mathrm{x} / 1024$ & $\mathrm{x} / 4096$ \\
\hline
\end{tabular}

The reduction of the number of iterations in the most computationally expensive step is a consequence of two advantages of the proposal. First of all, BP1 makes use of an empirical observation: most of the pixels that converge to correct values will normally do it in a low number of iterations. Thus, the number of iterations of the BP1 block can be very little. Secondly, after the pixel classification, the pixel data cost depends of the pixel type and this improves BP2 convergence. Additionally, BP2 only performs message passing over non-reliable pixels, reducing the number of iterations. Both contributions reduce the number of iterations and memory accesses but their computational impact is very limited.

\section{Occlusion, Edge Error and Texture-Less Area Handling}

In the RT-HD BP algorithm, the pixels are classified in 4 categories in the OE module: occluded, potential error, texture-less and reliable pixels.

The OE module generates the occlusion map using a cross-checking technique based on [10]. The module also detects low-textured areas by observing differences between the first ten minimum values on the fly. When the medium difference is bellow an experimental constant, the pixel is classify as texture-less.

In $\mathrm{BP}$, the disparity value for a given pixel is the label index that minimizes the sum of incoming messages and data-cost. When a pixel has converged in the BP algorithm, the sum of the incoming belief messages (SoIM function) tends to have a linear "V" shape (Figure 1.a). This shape is centered on the label index (disparity value). It has been empirically observed that the pixels that converge will normally present a SoIM function with a well defined " $V$ " shape during the first iterations of the last levels $(0,1)$ in the BP1 module, while the rest of the pixels normally present a non"V" shape or a SoIM function with several local minima (Figure 1.b).

Based on this observation, the proposed algorithm includes a simple technique to identify the pixels that probably converge. It is based on the comparison between the disparity label of the first and the third minimum. If the SoIM function has a "V" shape, the first (1M in figure 1$)$, second $(2 \mathrm{M})$ and third $(3 \mathrm{M})$ minimum disparity values will normally be consecutive values. However, if the shape is different, the third value will not normally be a consecutive value (Figure 1.b). This simple observation 
normally produces good results with a very low computational effort. The pixel whose SoIM function has a "V" shape will be classified as a reliable pixel and the rest are classified as potential error pixels. As occluded and textured-less pixels have previously been identified, the pixels that are classified as reliable have a high probability of having converged to the correct value.

The OE module generates a two-bit per pixel map that classifies the pixels in four categories: reliable, edge-error, occlusion and texture-less pixels.

\section{New Data Cost}

This module uses the information provided by $\mathrm{OE}$ to calculate new data costs as:

1. Reliable pixels: data cost defined as 0 for their minimum disparity label and a predefined penalty, equal to the maximum truncated value, for the rest labels.

2. Texture-less pixels: The data cost is 0 for all the disparity labels (unknown). This helps texture-less pixels to obtain correct disparity values.

3. Error pixels: they keep their data cost.

4. Occluded pixels: take the value of the first non-occluded pixel on their left.

BP2 limits the message passing to non-reliable pixels, reducing the memory traffic. The total memory reduction is about $21 \%$. This reduction is still far from the required 90\%. To reach this limit, a new improvement has been developed.

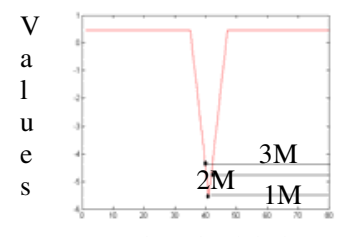

Disparity labels

a)

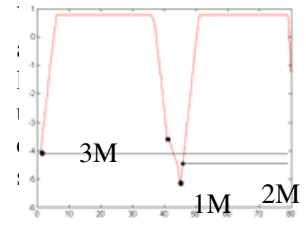

Disparity labels

b)

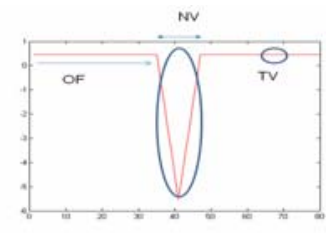

Disparity labels

c)

Fig. 1. a)Reliable pixel b) Possible error pixel c) Parameters for message compression

\section{Adaptive Message Compression}

The proposed compression method is based on the shape of the belief messages. Instead of storing only the envelope points, as in the EPG method in [18], the proposed technique stores all the points inside a region around the minimum disparity label. It has two main advantages with respect to [18]. Firstly, we do not need to uncompress the message prior to operate with it. Secondly, the compression rate drastically decreases when using EPG with limited precision. In contrast, we achieve more than $70 \%$ even with fixed point variables.

In our proposal, the number of stored points is a function of several parameters (adaptive approach): iteration, level and pixel type. This can reduce the compression factor, but increase the performance and reduce the quality penalty. This adaptive technique is applied only to the BP2 block reducing the memory traffic by about $70 \%$. The proposed compression technique stores 3 parameters and a set of points per message (Figure 1c): 
1. The offset (OF): first disparity label of the selected region.

2. Number of disparity labels (NV) of the selected region.

3. Information values: all the values of the selected region (from $\mathrm{OF}$ to $\mathrm{OF}+\mathrm{NV}$ ).

4. Truncating value (TV): value assigned to all the disparity labels outside the NV.

The pixels that converge will normally present a shape that is easily and efficiently compressed with the proposed techniques. This property, combined with the pixel classification that the OE generates, guarantees a good compression factor.

\section{Results}

In order to validate the proposed algorithms, several video sequences [21] have been evaluated with the classical and the RT-HD BP. In Fig 2, we show the disparity maps that are obtained with classical BP (a), the proposed RT-HD BP without compression (b) and the RT-HD BP with enough compression to meet the RT restrictions in section 2 (c). Some occlusions have been mitigated, some errors corrected and some texture-less zones have been filled in $(b, c)$.

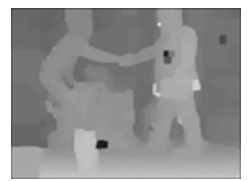

a)

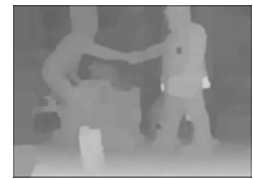

b)

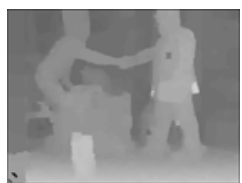

c)

Fig. 2. Disparity maps: a)Classical BP b) and c)RT-HD BP without and with compression

The RT-HD BP shows an improvement of more than $6 \%$ when compared to classical BP. At the same time, it satisfies section 2's RT and HD requirements. The memory reduction of the RT-HD BP with compression is about $90 \%$. To finish this results section, we provide Middlebury test results for our proposal in Figure 3 and Table 2, comparing the proposal with RT publications ranking Middlebury test. For convenience, we maintain the names used in Middlebury test in Table 2. As can be derived from Table 2, our proposal is the only ranking in the test that is able to achieve true RT for HD images. The only one whose latency is close to RT (RealtimeBP) works with small images. Moreover, between all of the proposals focus on real time, there is only one whose position in the ranking is significantly better than our proposal (PlaneFitBP) but working at $1 \mathrm{fps}$, which is not RT at all.

\begin{tabular}{|c|c|c|c|c|}
\hline AdaptPolygon [43] & 34.4 & 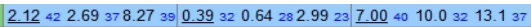 & $\underline{3.42} 33 \quad 10.0359 .0635$ & 5.81 \\
\hline RealTimeGPU [14] & 35.2 & 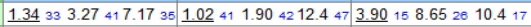 & 4.374210 .83912 .344 & 6.46 \\
\hline YOUR METHOD & 35.2 & $\underline{\underline{0.91}} 102.39$ з3 4.63 9 $\underline{\underline{0.66}} 361.37358 .7143 \underline{\underline{6.45}} 36 \quad 10.94015 .944$ & $\underline{\underline{6.14}} 48 \quad 13.64512 .343$ & 7.00 \\
\hline TensorVoting [9] & 35.3 & $1.20252 .18315 .8523 \quad \underline{0.68} 38 \quad 1.18346 .6938 \quad \underline{7.21} 44 \quad 14.446 \quad 17.549$ & 3.12279 .78329 .2036 & 6.58 \\
\hline GenModel [20] & 37.9 & 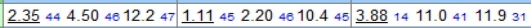 & $\underline{\underline{3.07}} 28 \quad 12.8448 .1028$ & 6.96 \\
\hline ReliabilityDP [13] & 39.8 & 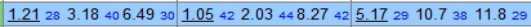 & $\underline{9.05} 5516.05114 .349$ & 7.44 \\
\hline $\mathrm{BP}+\mathrm{MLH}[40]$ & 40.0 & $\underline{1.62} 363.65438 .4140 \underline{\underline{0.66}} 371.87409 .0244 \underline{\underline{6.95}} 39 \begin{array}{ll}15.548 & 15.843\end{array}$ & $\underline{3.39} 32 \quad 13.7468 .5232$ & 7.43 \\
\hline
\end{tabular}

Fig. 3. Middlebury ranking for our proposal 
Table 2. Proposals focus on real-time, in middlebury ranking, comparison

\begin{tabular}{|c|c|c|c|c|}
\hline Parameter & Latency $(\mathrm{msec})$ & Image resolution & Disp. Lev. & Rank. \\
\hline Proposed RT-HD BP & 40 & $1280 \times 720$ & 80 & 35.2 \\
\hline RealTime GPU & 183 & $640 \times 480$ & 48 & 35.2 \\
\hline RTCensus & -- & -- & -- & 45.6 \\
\hline Realtime BP & $62.5^{*}$ & $320 \times 240$ & 16 & 30.5 \\
\hline FastAggreg & 600 & $450 \times 675$ & 60 & 32.7 \\
\hline PlaneFit BP & $1000^{*}$ & $512 \times 384$ & 48 & 12.8 \\
\hline
\end{tabular}

*Latency has been extrapolated from the fps data (18fps $\approx 62.5$ millisecond and $1 \mathrm{fps} \approx 1000$ milliseconds).

\section{Conclusions}

In this work we have presented a Real-Time High-Definition depth estimation algorithm based on Belief Propagation. It estimates depth maps in less than 40 milliseconds for HD images (1280x720 pixels at 30fps) with 80 disparity values. The work exploits the proposed double BP topology and it handles occlusions, potential errors and texture-less regions to improve the overall performance by more than a $6 \%$ (compared with classical BP) while it reduces the memory traffic by about $21 \%$. Moreover, the adaptive message compression method allows the system to satisfy Section-2's real-time and low execution latency requirements, reducing the number of memory accesses by more than a $70 \%$ with an almost negligible loss of performance (less than $0.5 \%$ ). The total memory traffic reduction is about $90 \%$ with a $6.0 \%$ performance improvement (compared with classical BP).

\section{References}

1. Vision project (2009), http: / /vision.tid.es

2. Diaz, J., Ros, E., Carrillo, R., Prieto, A.: Real-Time System for High-Image Resolution Disparity Estimation. In: IEEE TIP 2007 (2007)

3. Felzenszwalb, P.F., Huttenlocher, D.P.: Efficient belief propagation for early vision. In: IEEE CVPR 2004 (2004)

4. Hirschmuller, H.: Stereo Processing by Semiglobal Matching and Mutual Information. In: IEEE TPAMI 2008 (2008)

5. Yang, Q., Wang, L., Yang, R., Wang, S., Liao, M., Nister, D.: Real-time Global Stereo Matching Using Hierarchical Belief Propagation. In: BMCV 2006 (2006)

6. Wang, L., Liao, M., Gong, M., Yang, R., Nister, D.: High-quality real-time stereo using adaptive cost aggregation and dynamic programming. In: IEEE 3DPVT 2006 (2006)

7. Park, S., Jeong, H.: A fast and parallel belief computation structure for stereo matching. In: IASTED IMSA 2007 (2007)

8. Tseng, Y., Chang, N., Chang, T.: Low Memory Cost Block-Based Belief Propagation for Stereo Correspondence. In: IEEE ICME 2007 (2007)

9. Kolmogorov, V., Zabih, R.: Computing visual correspondence with occlusions using graph cuts. In: IEEE ICCV 2001 (2001)

10. Egnal, G., Wildes, R.: Detecting binocular half occlusions: empirical comparisons of five approaches. In: IEEE PAMI 2002 (2002) 
11. Sun, J., Li, Y., Kang, S., Shum, H.: Symmetric stereo matching for occlusion handling. In: IEEE CVPR 2005 (2005)

12. Yang, Q., Engels, C., Akbarzadeh, A.: Near Real-time Stereo for Weakly-Textured Scenes. In: BMCV 2008 (2008)

13. Gong, M., Yang, R.: Image-gradient-guided real-time stereo on graphics hardware. In: IEEE 3DIM 2005 (2005)

14. Yilei, Z., Minglun, G., Yee-Hong, Y.: Local stereo matching with 3D adaptive cost aggregation for slanted surface modeling and sub-pixel accuracy. In: IEEE ICPR 2008 (2008)

15. Yang, Q., Wang, L., Yang, R., Stewenius, H., Nister, D.: Stereo Matching with ColorWeighted Correlation, Hierarchical Belief Propagation, and Occlusion Handling. In: IEEE TPAML 2009 (2009)

16. Huq, S., Koschan, A., Abidi, B., Abidi, M.: Efficient BP Stereo with Automatic Parameter Estimation. In: IEEE ICIP 2008 (2008)

17. Sarkis, M., Diepold, M., Klaus: Sparse stereo matching using belief propagation. In: IEEE ICIP 2008 (2008)

18. Yu, T., Lin Super, R., Bei Tang, B.: Efficient Message Representations for Belief Propagation. In: IEEE ICCV 2007 (2007)

19. http://www . synplicty. com/products / haps / haps-52 .html (2009)

20. Liang, C., Cheng, C., Lai, Y., Chen, L., Chen, H.: Hardware-Efficient Belief Propagation. In: IEEE CVPR 2009 (2009)

21. Feldmann, M., Mueller, F., Zilly, R., Tanger, K., Mueller, A., Smolic, P., Kauff, T.: Wiegand HHI Test Material for 3D Video, MPEG 2008/M15413, Archans, Framce (April 2008) 